\title{
Comparison of Different Actuator Concepts for Applications in Rotating Machinery
}

\author{
HEINZ ULBRICH \\ Department of Mechanical Engineering, University of Essen, D-45117 Essen, Germany
}

\begin{abstract}
Considerable improvements of machines can be expected in the near future through the use of electronics in combination with control techniques. A key position will be held by the actuator systems that finally have to accomplish the controlled energy transfer according to the objectives. For rotating machine parts this is usually done via the bearings. Basically, there are two possibilities to transmit the controlled forces; contact free via magnetic fields (magnetic bearings) or the traditional way via conventional bearings. To realize the regulating actions multiple physical effects can be employed. In this paper some of the promising types of actuators and their advantages and disadvantages will be discussed, as well as how effectively to use these active elements under different objectives. Questions concerning the practical realizations will be given high priority.
\end{abstract}

Key Words: Rotors; piezo-; hydraulic- and magnetic actuators; transfer characteristics, modeling; control techniques

$\mathbf{T}$ he constant attempt to increase the productivity or efficiency of machines leads, in almost every field of mechanical and automotive engineering, to problems that cannot be solved by a mere adjustment of the construction parameters. Improvements of already-existing machines and vast innovations in new developments will be made possible by the use of electronics in combination with control techniques.

The heart of such new applications are the actuators. Depending on the objectives, they control the energy center of the system. In order to achieve this, some promising actuator concepts will be introduced and their strong and weak points will be discussed with regard to their industrial applications. The procedure of integrating the actuators into the whole system, the steering, and the design of an efficient control will be laid out. The objective of this paper is to give hints to help practically oriented engineers choose the appropriate actuators for their specific application.

\section{POSSIBLE ACTUATORS}

In the future, the dynamics of machines will be specifically and purposely adapted to the requirements of the particu- lar technical task by the use of electronics in combination with control techniques. A key position will thereby be held by the actuators, which will perform the energy transfer, depending on the objective. The actuators have the task of transforming the information about the systemsupplied by the sensors-into the desired physical actions. To realize these regulating actions, multiple and also very different physical effects can be used. With regard to industrial applications, the following demands-as well as the costs—should be given high priority:

- compactness, because usually the available space is quite limited

- large forces, which has to be seen in relation to the masses to be moved and the necessary regulating distances, as well as the regulating frequencies to be realized

- regulating frequencies at least as high as the highest vibration to be controlled

- simple actuator transfer characteristics to avoid additional dynamic problems due to the actuator dynamic

- light weight, which is a major criterion for applications in aeronautics and the space industry

In what follows some actuator concepts will be discussed and commented upon regarding their advantages and disadvantages. 


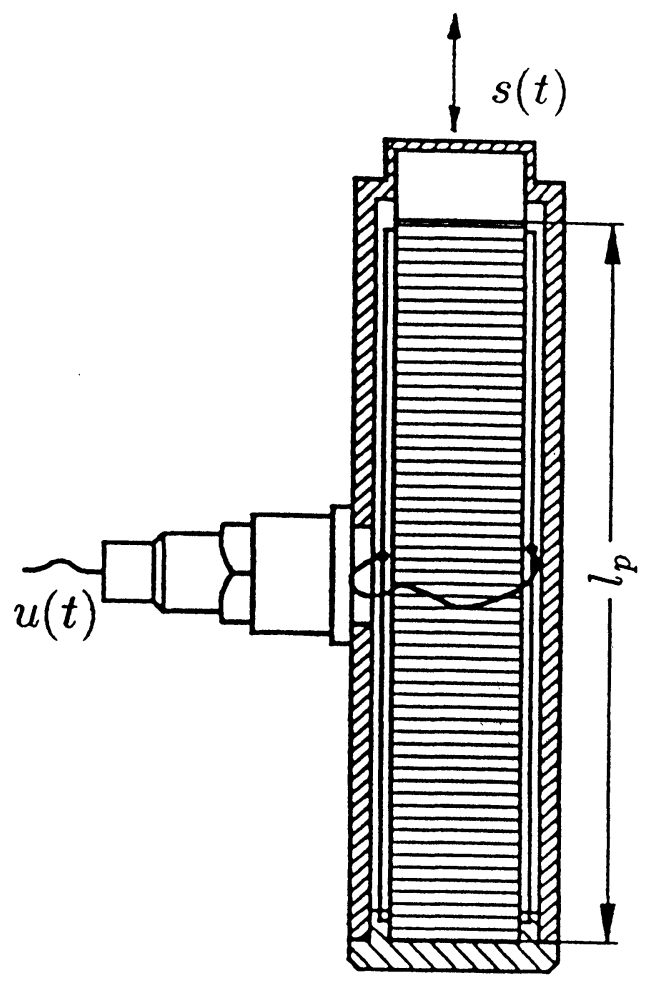

FIGURE 1 Schematic of piezo actuator.

\section{Piezo Actuators}

Piezo actuators have been commonly used for some time. They are especially well suited when dealing with vibrations with small amplitudes or to adjust mirror systems or other components in high-precision constructions within the $\mu \mathrm{m}$-range (Haidenwanger and Klose [1992]; Goto [1992]). They have also been investigated theoretically and experimentally for their ability to influence rotors via conventional bearings (Palazzolo [1989], [1992]; Santos and Ulbrich [1992]) and mechanisms in general (Liao and Sung [1990]). Piezo actuators' special merits are their high stiffness $k_{p}$ and their performance in highfrequency ranges. The realizable control forces can be calculated by

$$
f_{p}(t)=I_{p} C_{p} k_{p} u(t)-k_{p} s(t)
$$

where $I_{p}$ is the piled height of the crystal wafers, $C_{p}$ is the electrostatic capacity (each crystal wafer is a capacitor), and $k_{p}$ is the stiffness of the actuator (see Figure 1). The regulating distance is called $s(t)$, the control voltage $u(t)$.

The crucial disadvantages that limit the application range are the small, realizable, regulating distances $\left(\approx I_{p} / 1000\right)$ and the very low material damping of the actuators (impacts are transfered almost undamped). Due to the dependency of the regulating distance on the total height, the size of piezoelectric actuators can be quite large. Also the permissable compressive load is very small so usually the control forces can only be applied through additional transfer elements (Palazzolo [1989]). A typical transfer function of a piezo actuator is shown in Figure 2. It can be seen that a strong reduction of amplitude begins at $200 \mathrm{~Hz}$ and the phase lag progresses linearly with the frequency. At $1000 \mathrm{~Hz}$ the phase lag is already $-180^{\circ}$.

\section{Hydraulic Actuators}

Figure 3 shows a sectional view of a newly developed hydraulic actuator. The operating part consists of two cylindrical chambers which are closed in the working direction (in Figure 3 the vertical direction) by elastic membranes. The pressure difference $\Delta p$ between the two chambers 1 and 2, necessary to create the regulating operation, is supplied by the servo valve.

The special design of the actuator yields a high radial stiffness and ensures a corresponding safety against tilting effects (depending on the distance between the two membranes as well as on their thickness). Through this arrangement the regulating movements can be applied completely without friction.

The length $I_{H}$ of the hydraulic actuator is changed by the length $s(t)$ due to the regulating action. The control force can be expressed by an equation similar to that of the piezo system:

$$
f_{H}(t)=A^{*} \Delta p(t)-k_{M} s(t)
$$

where $A^{*}$ is the characteristic membrane area, $k_{M}=2 c_{L}$ the effective membrane stiffness, $c_{L}$ the membrane stiffness, and $s(t)$ the regulating distance in the direction of the force. The regulating force is generated by the pressure difference $\Delta p$ and not (different from the piezo actuator system) proportional to the control voltage. Unfortunately, the regulating pressure strongly depends on all fluidmechanical losses of the hydraulic and the dynamic of the servo valve. Thus the realizable regulating frequencies are mainly determined by the cut-off frequency of the used servo valve and some hydrodynamic influences (pipes, fluid). Due to these hydrodynamic influences the actuator has basically an integrating transfer characteristics, as can be seen in Figure 4 (Althaus and Ulbrich [1992]).

Due to the choice of membranes (diameter, thickness) a multitude of static characteristic curves can be realized. Also, the maximum control forces and regulating distances can be adapted in wide ranges with an adequate choice of the membranes. The given design limit is al- 


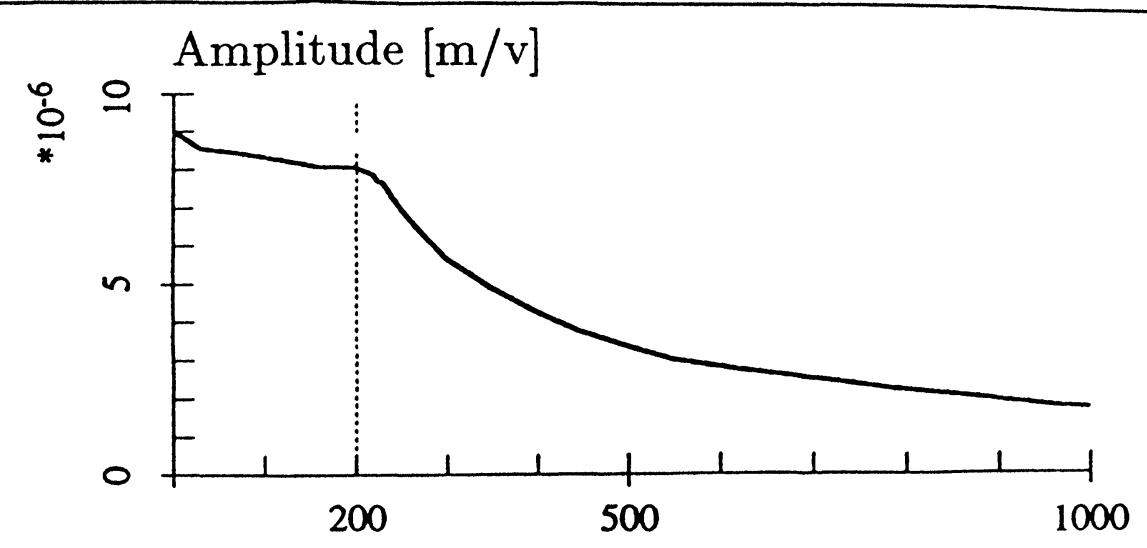

$[\mathrm{Hz}]$

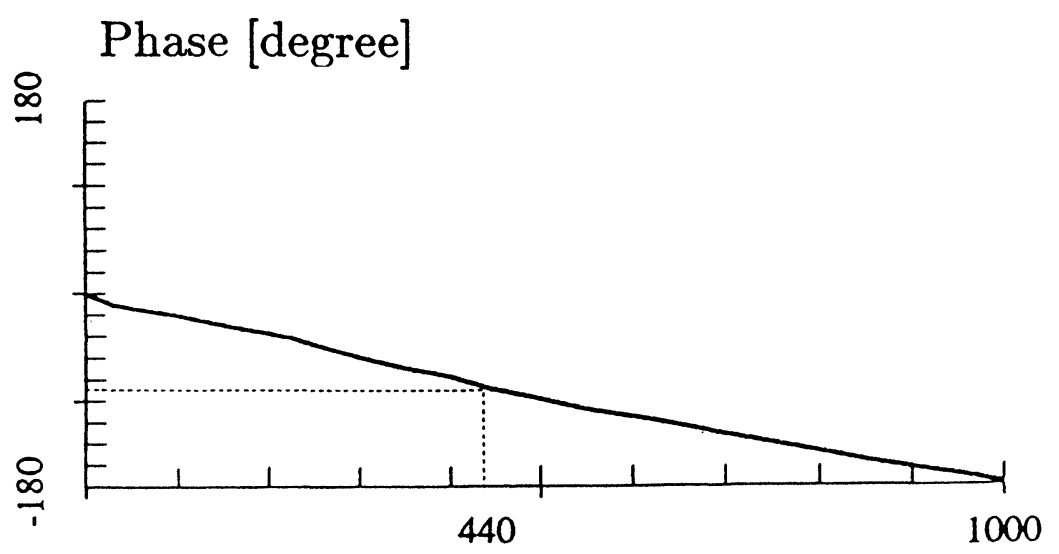

$[\mathrm{Hz}]$

FIGURE 2 Measured transfer function of the investigated piezo actuator (ratio regulating distance $s(t)$ over input voltage $u(t)$ ) (Santos [1993]).

ways the maximum material stress in the membranes. The thicker the membranes at a given diameter, the larger the stiffness of the actuator and the smaller the maximum regulating distance. For practical purposes this is not a problem because the generally high stiffnesses of the systems under consideration allow only small deformations and thus require only small regulations. In this context it should also be noted that the physics comply with the regulating actions since in the deflected state the controller generally has to apply resilient forces in opposite direction to the deflection $s(t)$. This means the term $k_{H} s(t)$ from eq. (2) will be superimposed by the control force $A^{*} \Delta p(t)$. A typical transfer function of a hydraulic actuator is shown in Figure 5.

When using these actuators to control rotor vibrations, two actuators with radial working directions can be positioned perpendicular to each other if necessary (Ulbrich [1992]).

\section{Magnetic Actuators}

Magnetic actuators can be classified into two types. One type are the magnetic bearings that permit a non-contact force transfer. The other type are the electromagnetic actuator systems where the regulating actions - as with the other presented actuator concepts-are applied indirectly, usually via conventional bearings. In both types the magnetic forces can be controlled by either current or voltage. In the case of control by current (the power amplifiers are working as a current source) the relationship between the realizable force $f(t)$, the control current $i(t)$ and the reg- 


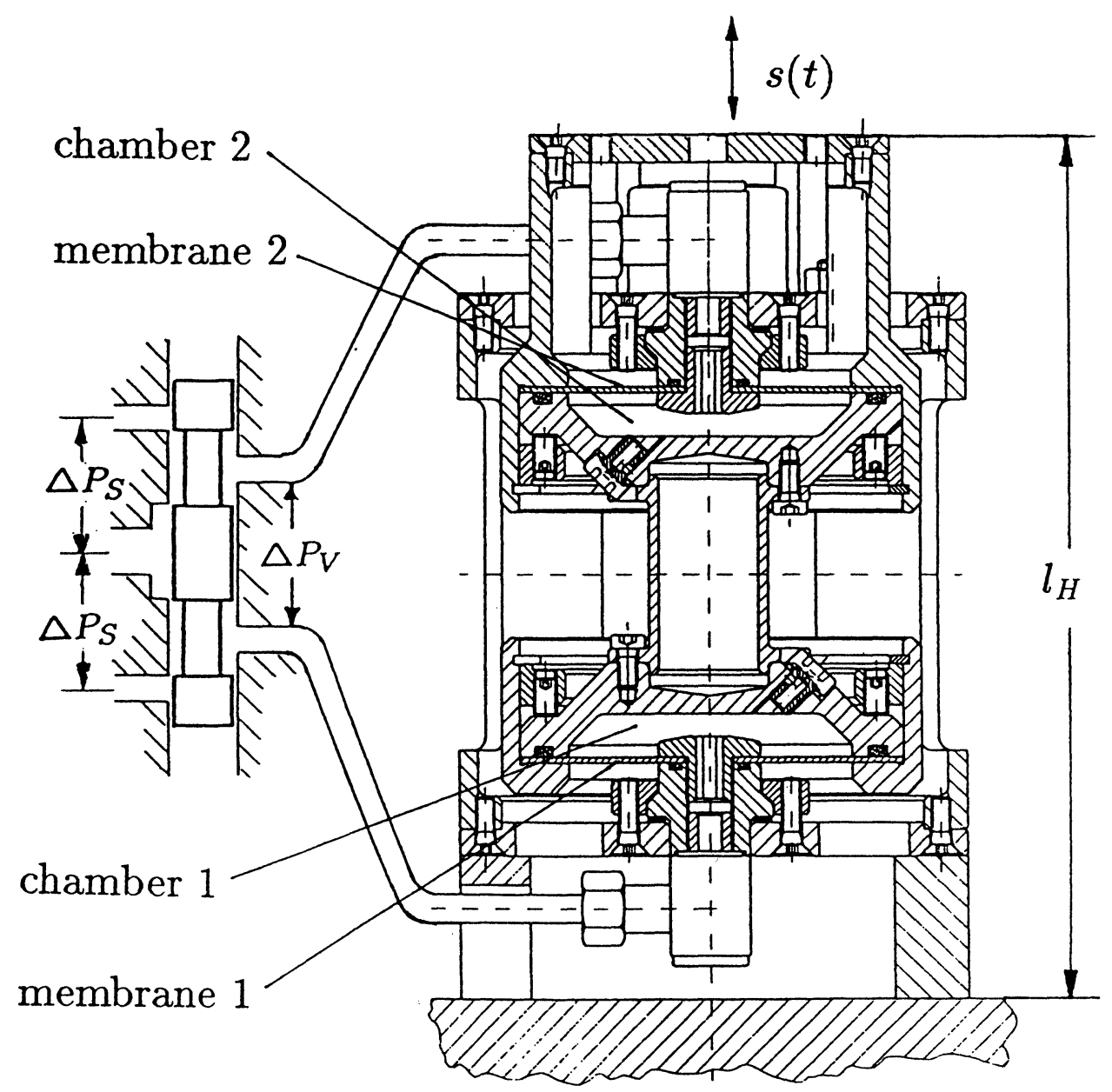

FIGURE 3 Hydraulic actuator.

ulating distance $s(t)$ is strongly nonlinear. The resulting force can be expressed by

$$
\begin{aligned}
f(t)=\frac{s_{0}^{4}}{\left(s_{0}^{2}-s^{2}\right)^{2}} & {\left[k_{i} i+\left(k_{s}-k_{M}\right) s+\frac{k_{s}}{k_{W} i_{0}^{2} s_{0}}\right.} \\
& \left.\times\left(k_{W} i_{0} i s^{2}+s_{0} s i^{2}\right)\right]
\end{aligned}
$$

where $s_{0}$ is the air gap in neutral position, $i_{0}$ is the constant premagnetizing current, and $k_{i}, k_{s}, k_{W}$ and $k_{M}$ are constants depending mainly on the geometry of the actuator design, the used magnetic material and the employed electrical components. Under the assumptions of small control currents $i(t) \ll i_{0}$ and small regulating distances $s(t) \ll s_{0}$ the following linear correlation between the control current and the resulting force can be obtained:

$$
f(t)=k_{i} i(t)+\left(k_{s}-k_{M}\right) s(t)
$$

The constants $k_{i}$ (force-current coefficient), $k_{s}$ (forcedisplacement coefficient) and $k_{M}=2 c_{L}$ (stiffness factor of the assembly in working direction, $c_{L}$ stiffness of the membranes) permit large varieties in layout and construction of the actuators. Equation 4 shows the destabilizing effect of such a magnetic actuator system due to a negative stiffness indicated by the $+k_{s}$ factor. The most severe case of this destabilizing effect occurs in non-contact magnetic bearings $\left(k_{M}=0\right.$ !).

Figure 6 shows two types of magnetic actuator systems that are currently being developed. The difference between both types is basically the realization of the premagnetizing. In type $\mathrm{I}$, the necessary premagnetizing is realized electrically (indirectly) and in type II, it is realized by special permanent magnets (directly). The magnetic potential is realized in type I through the product $i_{0} w$ ( $i_{0}$ is the premagnetizing current, $w$ is the number of windings in the premagnetizing coil) and in type II the circulation $i_{0} w$ with 


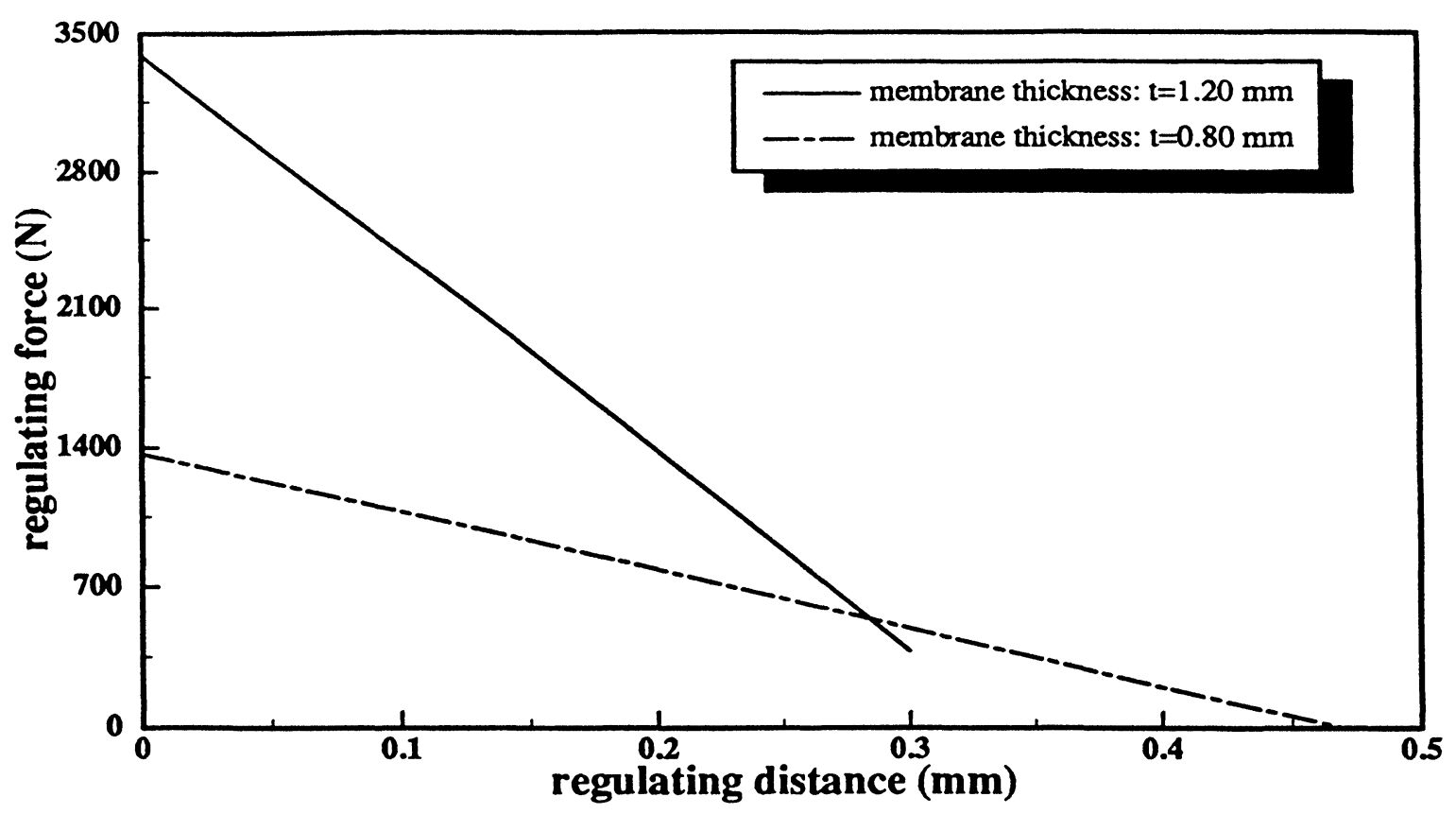

FIGURE 4 Force-distance relations for the hydraulic actuator.
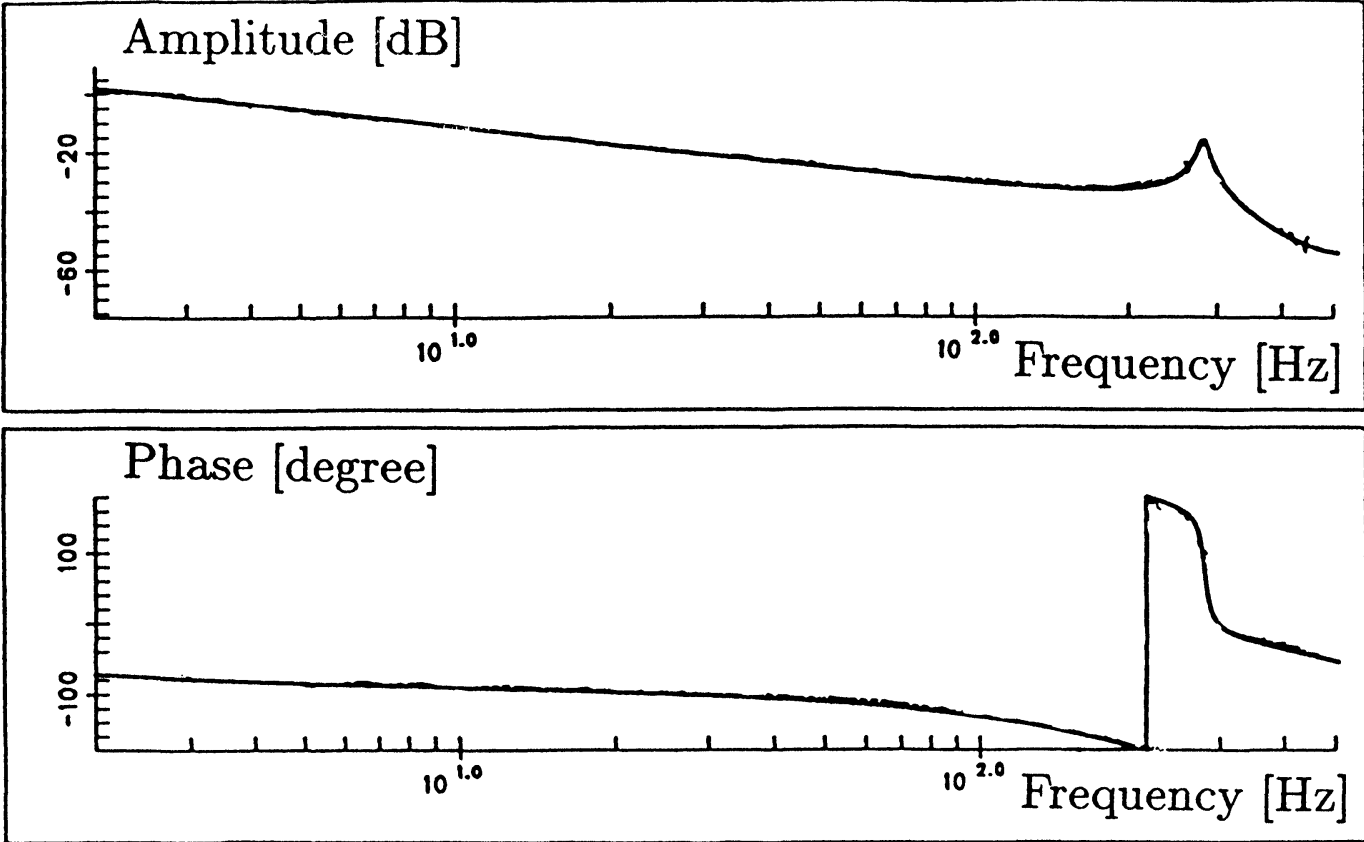

FIGURE 5 Measured transfer characteristic of an investigated hydraulic actuator (ratio regulating distance $s(t)$ over input voltage to servo valve $u(t)$ ) (Althaus and Ulbrich [1992]). 
electromagnetic actuator (type I)

(pre-magnetizing with electromagnet)

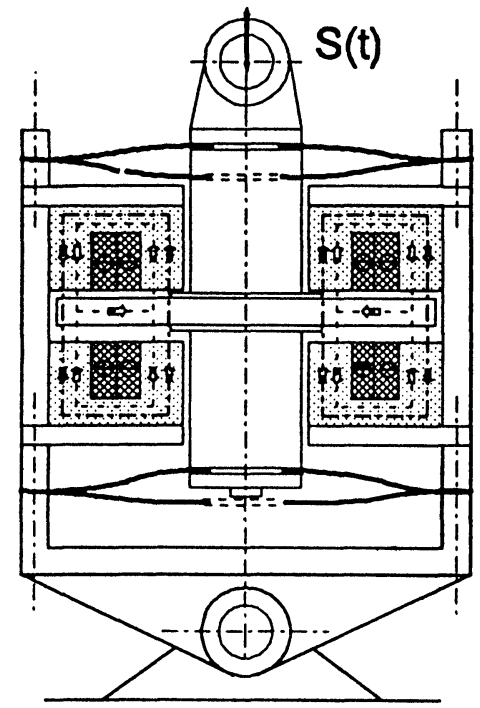

electromagnetic actuator (type II)

(pre-magnetizing with permanent magnet)

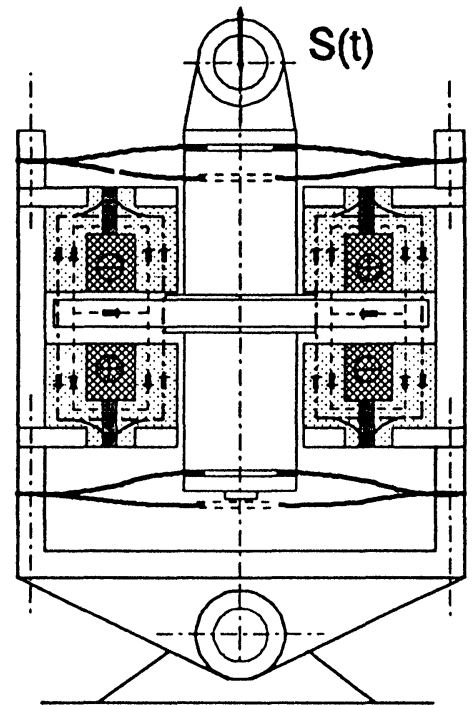

FIGURE 6 Comparison of different magnetic actuator concepts

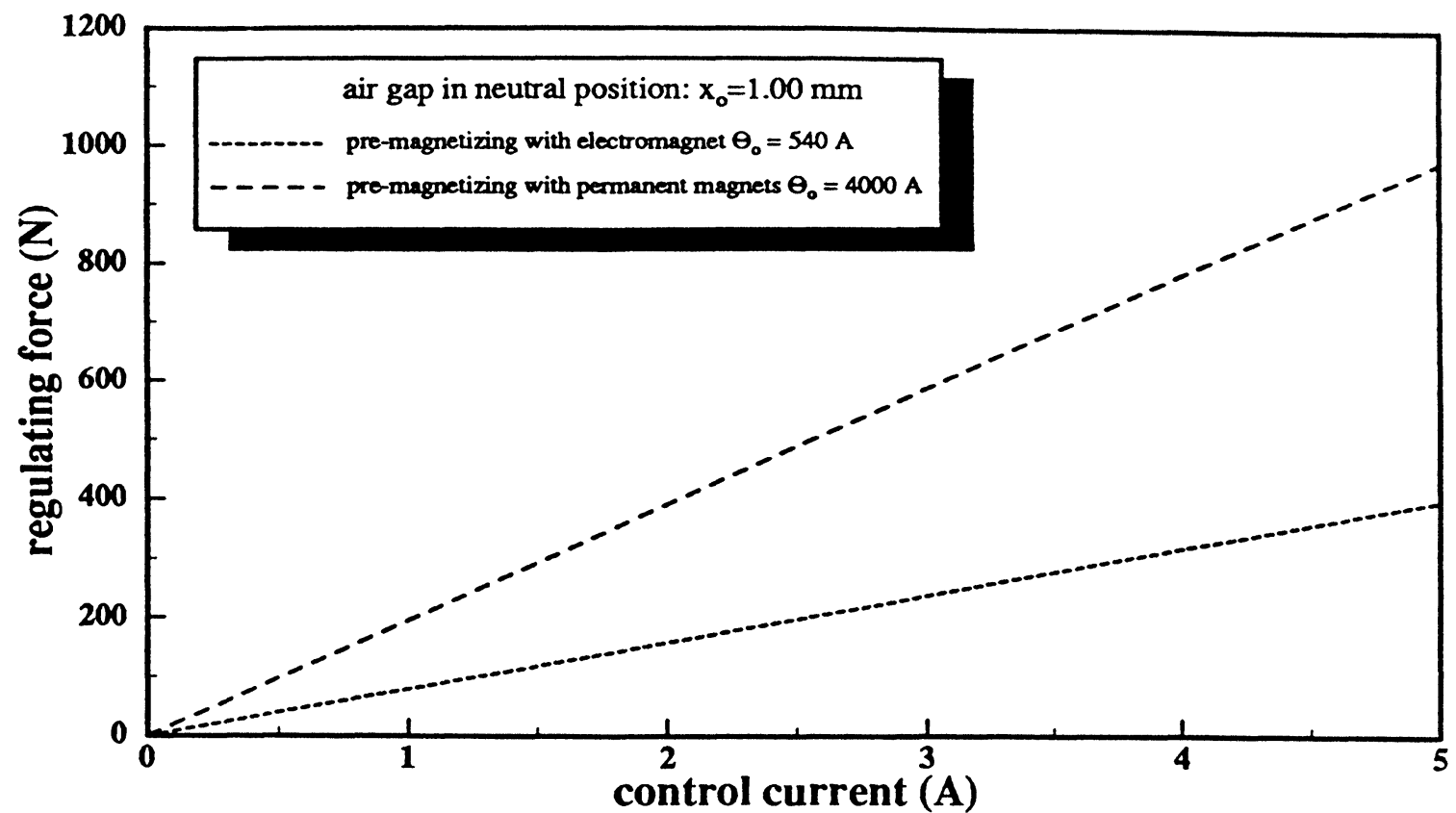

FIGURE 7 Comparison of the regulating forces in magnetic actuators as a function of the control current (electrical premagnetizing-, permanent magnet premagnetizing -). 
respect to the premagnetizing is realized by the integrated permanent magnets (magnetic potential $\Theta_{\mathrm{pm}} \triangleq i_{0} w$ ). Figure 7 shows the control force as a function of the control current for both types. Tuning of both systems with the objective of maximizing the control forces at a constant actuator size shows that using permanent magnets for the premagnetizing results into a control force about 2.5 times as high as in the case of indirect premagnetizing.

Figure 8 shows the measured control force frequency curve for type I up to a frequency of $300 \mathrm{~Hz}$. It turns out that the phase lag at $300 \mathrm{~Hz}$ is already $-60^{\circ}$. Substantial improvements can be expected here by the use of new soft-magnetic materials. These experimental investigations have not yet been finished.

\section{SUMMARY}

Figure 9 gives an overview of the discussed actuator systems with regard to their realizable regulating distances and frequencies. Table I shows a rating of some characteristics of the different actuator systems with regard to their specific requirements. This should just be understood as a rough classification, but it already allows to make out the typical applications of all the discussed actuator systems. New developments in the field of magnetic materials let us expect an even stronger shifting of their attributes toward magnetic actuators (Meeks [1992]; O’Connor [1992]).

\section{INCLUDING THE ACTUATORS INTO THE SYSTEM TO BE CONTROLLED}

An efficient use of actuators requires a mathematical description of the open-loop system that has been adapted to the problem and where the actuators, which are also represented by their mathematical models, have been integrated. The resulting model is able to describe the physical relations of the complete dynamic interactions between the open-loop system and the actuators.

\section{Mathematical Description of the System}

General movements of systems consisting of rigid, elastic, and fluid components can be mathematically described by a system of differential equations (Bremer [1989]):

$$
\mathbf{M}(\mathbf{q}, t) \ddot{\mathbf{q}}+\mathbf{f}(\mathbf{q}, \dot{\mathbf{q}}, t)=\mathbf{J}^{T}(\mathbf{q}) \mathbf{h}_{\text {controller }}(t)=\mathbf{b}(\mathbf{q}, t)
$$

Vector $\mathbf{q}(t)$ represents the generalized coordinates that describe the movements of the system. The mass matrix $\mathbf{M}(\mathbf{q}, t)$ generally depends on $\mathbf{q}$ and explicitly the time $t$. Vector $\mathbf{f}(\mathbf{q}, \dot{\mathbf{q}}, t)$ considers all the forces involved in the energy household (except the control forces). Vector $\mathbf{b}(\mathbf{q}, t)$ contains the forces resulting from the steering and control whose components act in the direction of the generalized coordinates (this results immediately from the multiplication of $\mathbf{h}(t)$ with the Jacobian matrix $\left.\mathbf{J}^{T}\right)$. Closed kinematic loops can be treated with this method as well as elastic components (e.g., in actively controlled four-bar linkages; Ulbrich and Ahlemeyer [1994]). For the design of the control it is important whether the geometrical parameters (e.g., the distance $s(t)$ may be given as a forced condition) or the forces (an additional degree of freedom will be introduced if not already included) are controlled.

In general, a reference movement, for example, the rigid body motion of a function-generating mechanism or a flight maneuver of an airplane turbine-is given by the vector $\mathbf{q}(t)$. The nonlinear equation of motion for this reference movement can be gained from eq. 5 :

$$
\mathbf{M}_{r}\left(\mathbf{q}_{r}\right) \ddot{\mathbf{q}}_{r}+\mathbf{g}\left(\mathbf{q}_{r}, \dot{\mathbf{q}}_{r}\right)=\mathbf{b}_{r}(t)=\mathbf{u}_{r}(t)
$$

With a given $\mathbf{q}_{r}(t), \dot{\mathbf{q}}_{r}(t)$ and $\ddot{\mathbf{q}}_{r}(t)$ the steering vector $\mathbf{u}_{r}(t)$ to realize this reference movement can now directly be calculated. Given the case that undesired small superimposed deviations appear-represented by the vector $\mathbf{e}(t)$ - that are composed of small rigid body movements or also possibly elastic deformations of the structure, a control can be added. The starting point for the design of such a control is the linearized system (linearized around the reference movement, i.e. $\mathbf{q}(t)=\mathbf{q}_{r}(t)+\mathbf{e}(t),|\mathbf{e}| \ll\left|\mathbf{q}_{r}\right|$, eq. 4):

$$
\mathbf{M}_{e}(t) \ddot{\mathbf{e}}(t)+\mathbf{P}_{e}(t) \dot{\mathbf{e}}(t)+\mathbf{Q}_{e}(t) \mathbf{e}(t)=\mathbf{b}_{e}(t)=\mathbf{u}_{e}(t)
$$

\section{Comments on the Control}

The realization of an efficient control will be decisively influenced by the choice of the actuator- and sensor systems and their positioning within the whole system as well as the choice of the control concepts including the optimization of the controller. The optimization of the controller strongly depends on the chosen objectives. In any case the controller will be optimized with respect to a criterion for the quality of the employed control. Discussions of different quality criteria and some remarks on the further procedure can be found in Bremer [1990] and Ulbrich [1992].

For the optimization of controllers there are already a multitude of computer programs available that usually result into a parameter optimization, that is, tuning of the 


\section{Amplitude $[\mathrm{dB}]$}

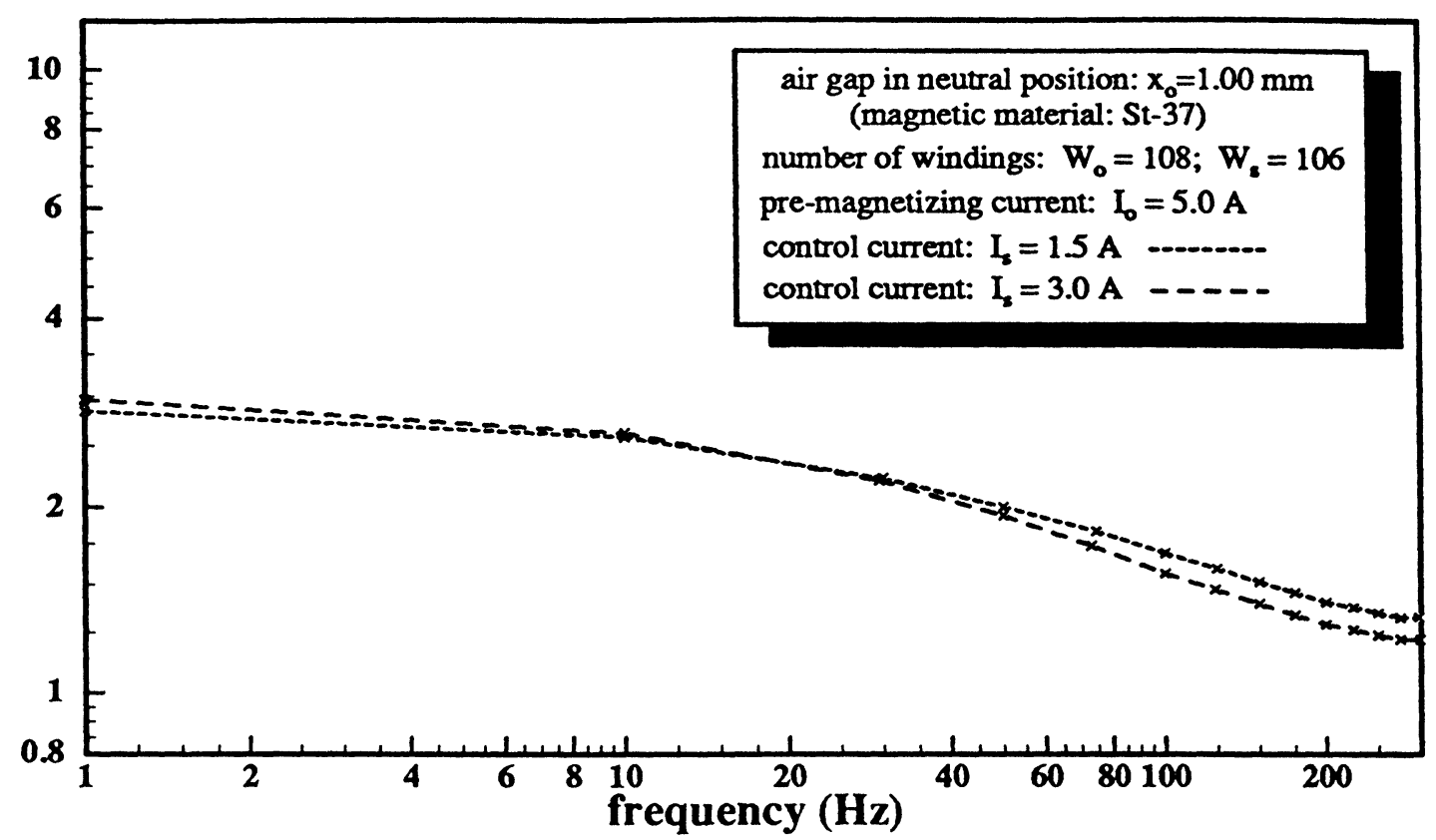

Phase [deg]

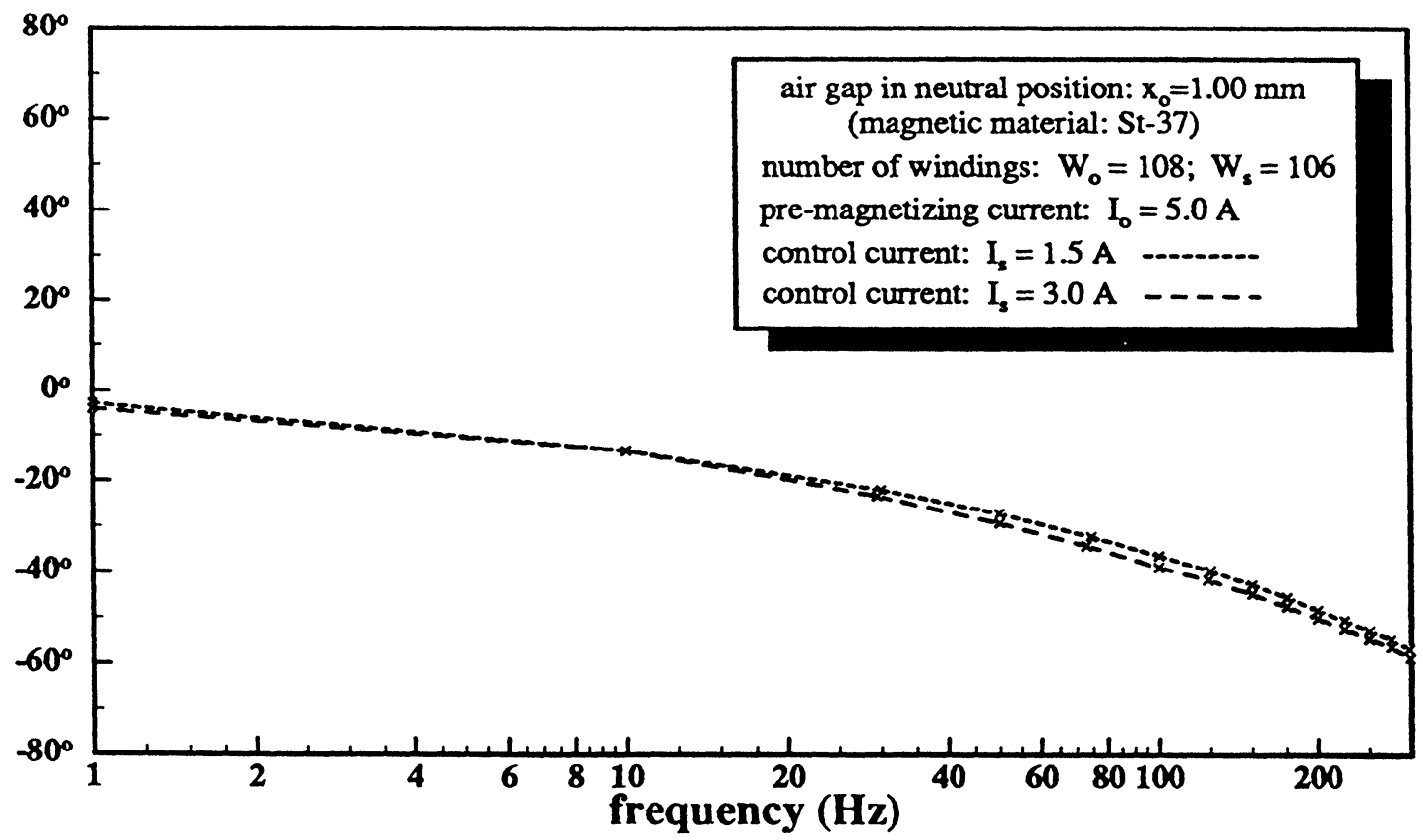

FIGURE 8 Transfer characteristic of the magnetic actuator system with electrical premagnetizing and ordinary magnetic material (Steel ST37). 


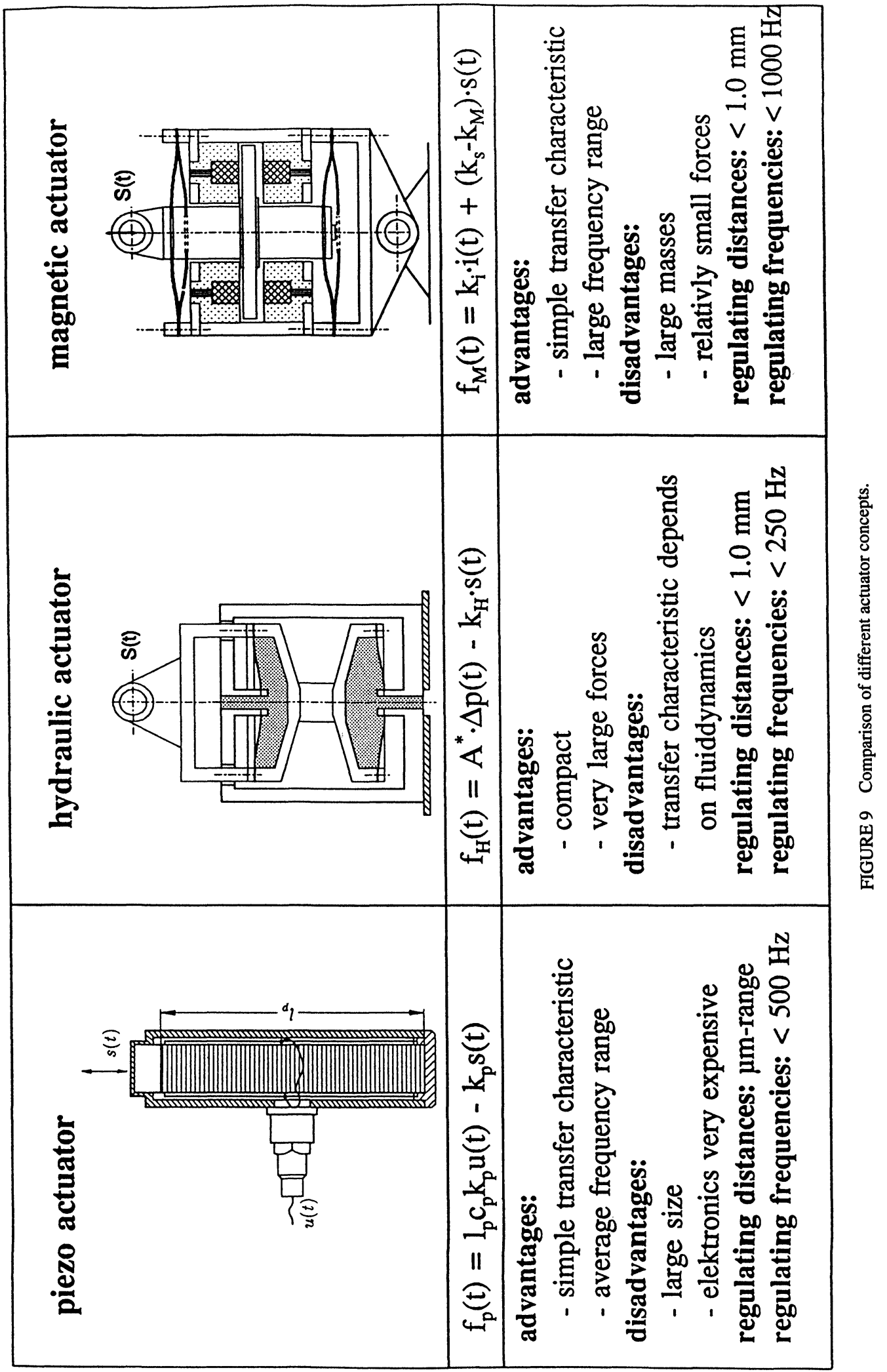


TABLE I

Rating of different actuator concepts

\begin{tabular}{|c|c|c|c|}
\hline \multirow[b]{2}{*}{ Characteristic } & \multicolumn{3}{|c|}{ Actuator Type } \\
\hline & Electromagnetic & Piezoelectric & Hydraulic \\
\hline Frequency range & +++ & +++ & + \\
\hline Regulating distance & ++ & - & $++t$ \\
\hline Stiffness & + & +++ & ++ \\
\hline $\begin{array}{l}\text { Realizable forces/weight } \\
\text { (active element without periphery) }\end{array}$ & + & + & +++ \\
\hline Realizable forces/size & + & - & +++ \\
\hline $\begin{array}{l}\text { Realizable forces/total weight } \\
\text { (including periphery, e.g., hydraulics) }\end{array}$ & +++ & ++ & - \\
\hline $\begin{array}{l}\text { Possibility of stimulation of vibrations } \\
\text { due to nonlinearities }\end{array}$ & ++ & + & - \\
\hline
\end{tabular}

\begin{tabular}{lccc}
\hline & Applying the Actuators for the Control of High-Speed Rotors & \\
\hline Characteristic & Electromagnetic & Piezoelectric & Hydraulic \\
\hline Requirements against wear & +++ & - & - \\
Power loss (e.g., due to bearing friction) & +++ & - & - \\
Temperature range & +++ & + & - \\
Cleanliness & ++ & + & + \\
Emergency operation characteristics & +++ & & + \\
Longevity or life & & & +
\end{tabular}

controller amplifications to optimize the quality criterion to obtain an optimal system performance according to the objectives.

The realization of the control can be analog, digital, or "hybrid". An analog control will, in most cases, not be flexible enough and is only employed in so-called constant controllers. When system changes during the operation are considered or nonlinear controllers are realized then usually the advantages of a digital control can be exploited. The only problem that can arise in digital controllers is the limited cycle time, but that depends on the particular task to be solved. Also, new developments in the field of parallel computer design (transputer) will further reduce the cycle times and can as such constitute a solution. If cycle times are still not fast enough it can sometimes be useful to employ a "hybrid control" in order to separate the time-critical components, for example, the rotorfrequent vibrations of high-speed rotors (Ulbrich [1982]), and to combine a fast analog control with a digital adaptation of the control parameters.

\section{CONCLUSION}

The advance of electronics in combination with automatic control technology will continue. Through constantly in- creasing the speed of computers as well as improving the precision of analog elements, more and more complicated calculations and simulations, embedded into the information circuits of controlled systems, will contribute to improve further the dynamics of these systems. It is just a matter of time before active bearings will be regularly employed in machine tools, gas turbines, terrestrial turbines, engine suspensions, valve control, and various other mechanisms. To account for these future requirements a practically oriented development of actuators that are specifically adapted to the particular problem with a simultaneous integration of the necessary sensors is of high importance. To realize this, some concepts that are partially still under development have been presented and discussed and the task of how to come to an efficient use of such active components to improve machines in their dynamic characteristics, has been explained.

$\begin{array}{ll}\text { Nomenclature } \\ \mathbf{b}(\mathbf{q}, t) & \text { vector of generalized control forces } \\ \mathbf{b}_{r}(t) & \text { control vector for realizing the reference motion } \\ \mathbf{e}(t) & \text { error vector considering the deviation from reference } \\ \mathbf{f}(\mathbf{q}, \dot{\mathbf{q}}, t) & \text { motion } \\ f_{H}(t) & \text { vector of generalized forces } \\ f_{M}(t) & \text { control force of hydraulic actuator [N] } \\ f_{p}(t) & \text { control force of magnetic actuator [N] } \\ \mathbf{g}\left(\mathbf{q}_{r}, \dot{\mathbf{q}}_{r}\right) & \text { gentrol force of piezo actuator [N] }\end{array}$


$i(t) \quad$ control current of magnetic actuator [A]

$k_{H} \quad$ effective membrane stiffness of hydraulic actuator $[\mathrm{N} / \mathrm{m}]$

$k_{i} \quad$ force-current coefficient [N/A]

$k_{M} \quad$ membrane stiffness of magnetic actuator $[\mathrm{N} / \mathrm{m}]$

$k_{p} \quad$ stiffness of piezo actuator $[\mathrm{N} / \mathrm{m}]$

$k_{s} \quad$ force-displacement coefficient $[\mathrm{N} / \mathrm{m}]$

$I_{p} \quad$ length of piezo actuator $[\mathrm{m}]$

$\Delta p(t) \quad$ control pressure [Pa]

$\mathbf{q}(t) \quad$ vector of generalized coordinates

$\mathbf{q}_{r}(t) \quad$ vector of generalized coordinates of the linearized coordinates

$s(t) \quad$ regulating distance $[\mathrm{m}]$

$\mathbf{u}(t) \quad$ input voltage of the piezo actuator [V]

$\mathbf{u}_{e}(t) \quad$ control vector to minimize the error $\mathbf{e}(t)[\mathrm{N}]$

$A^{*} \quad$ effective membrane area $\left[\mathrm{m}^{2}\right]$

$C_{p} \quad$ capacity of the piezo actuator $[\mathrm{F}]$

$\mathbf{J}_{q}(\mathbf{q}) \quad$ Jacobian matrix

$\mathbf{M}(\mathbf{q}, t) \quad$ mass matrix

$\mathbf{M}_{e}(t) \quad$ mass matrix of error system

$\mathbf{M}_{r}\left(\mathbf{q}_{r}\right) \quad$ mass matrix of reference system

$\mathbf{P}_{\rho}(t) \quad$ damping matrix of error system

$\mathbf{Q}_{e}(t) \quad$ stiffness matrix of error system

\section{References}

Althaus, J., and Ulbrich, H., 1992. A Fast Hydraulic Actuator for Active Vibration Control, Proceedings of the Institute of Mechanical Engineers, International Conference "Vibrations in Rotating Machinery," Bath, England.

Bremer, 1989. Dynamik und Regelung Mechanischer Systeme, B.G. Teubner, Stuttgart.

Goto, H. et al., 1992. Miniature 2-Dimensional Optical Scanner Utilizing a Multilayered Piezoelectric Actuator with New Displacement Expan- sion Mechanism, Proceedings of the 3rd International Conference on New Actuators, Bremen, Germany June 24-26.

Haidenwanger, H.-G. and Klose, P. L. M., 1992. 004 Isolation and Compensation of Vibration by Means of Active Piezo-Ceramic Mounts, International Symp. on Advanced Vehicle Control, AVEG 92, Japan.

Liao and Sung, 1990. An Elastodynamic Analysis and Control of Flexible Linkages Using Piezoceramic Sensors and Actuators, ASME Des Eng Div Pub, DE v 24, Flexible Mechanisms, Dynamics and Robot Trajectories, 21st Biennial Mechanism Conference, Chicago. ASME, New York pp. 503-510.

Meeks, R., 1992. Magnetic Bearings Boost Performance, Global Gas Turbine News.

O'Connor, L., 1992. Active Magnetic Bearings Give Systems a Lift, Journal of Mechanical Engineering.

Palazzolo, A. B. et al., 1989. Piezoelectric Pushers for Active Vibration Control of Rotating Machinery, Transactions of the ASME, Journal of Vibration, Acoustics, Stress, Reliability in Design, Vol. 111, pp. 298-305.

Palazzolo, A. B. et al., 1992. Hybrid Active Vibration Control of Rotor Bearing Systems Using Piezoelectric Actuators, Transactions of the ASME, Journal of Vibration and Acoustics.

Santos, I., and Ulbrich, H., 1992. Actively Controlled Tilting Pad Journal Bearings Fourth International Symposium on Transport Phenomena and Dynamical Machinery, Honolulu, HI.

Santos, I., 1993. Aktive Kippsegmentlagerung-Theorie and Experiment, Dissertation, VDI-Fortschritberichte, Reihe 11, Nr. 189, Düsseldorf.

Ulbrich, H., 1992. Active Bearing Support for Rotating Machine Elements, Journal of Machine Vibration, Springer-Verlag, London.

Ulbrich, H., and Ahlemeyer, E., 1994. Präzise Bewegung nichtlinearer Übertragungsgetriebe durch aktive Elemente, ZAMM, Nr. 74, 4, pp. T81-T88. 

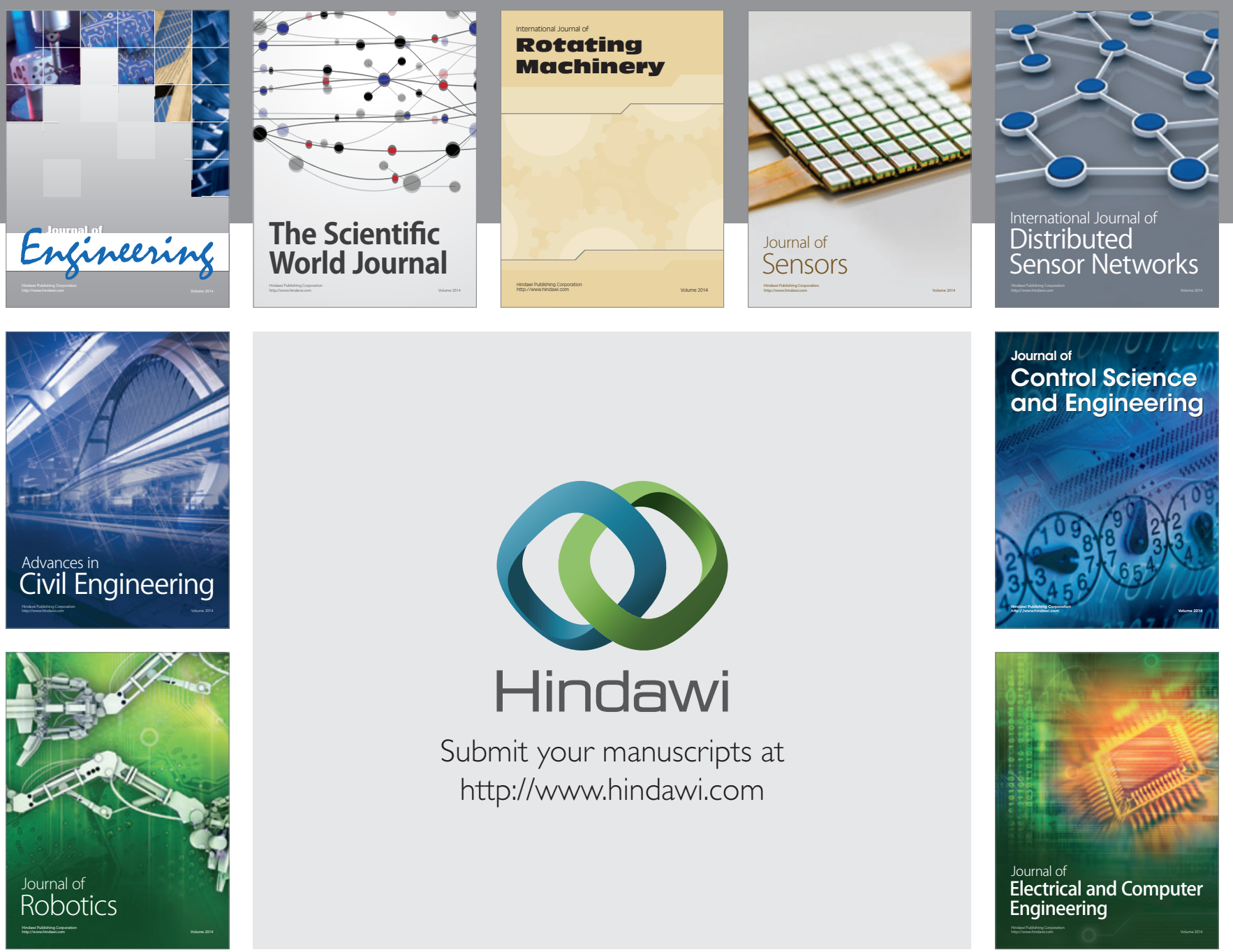

Submit your manuscripts at

http://www.hindawi.com
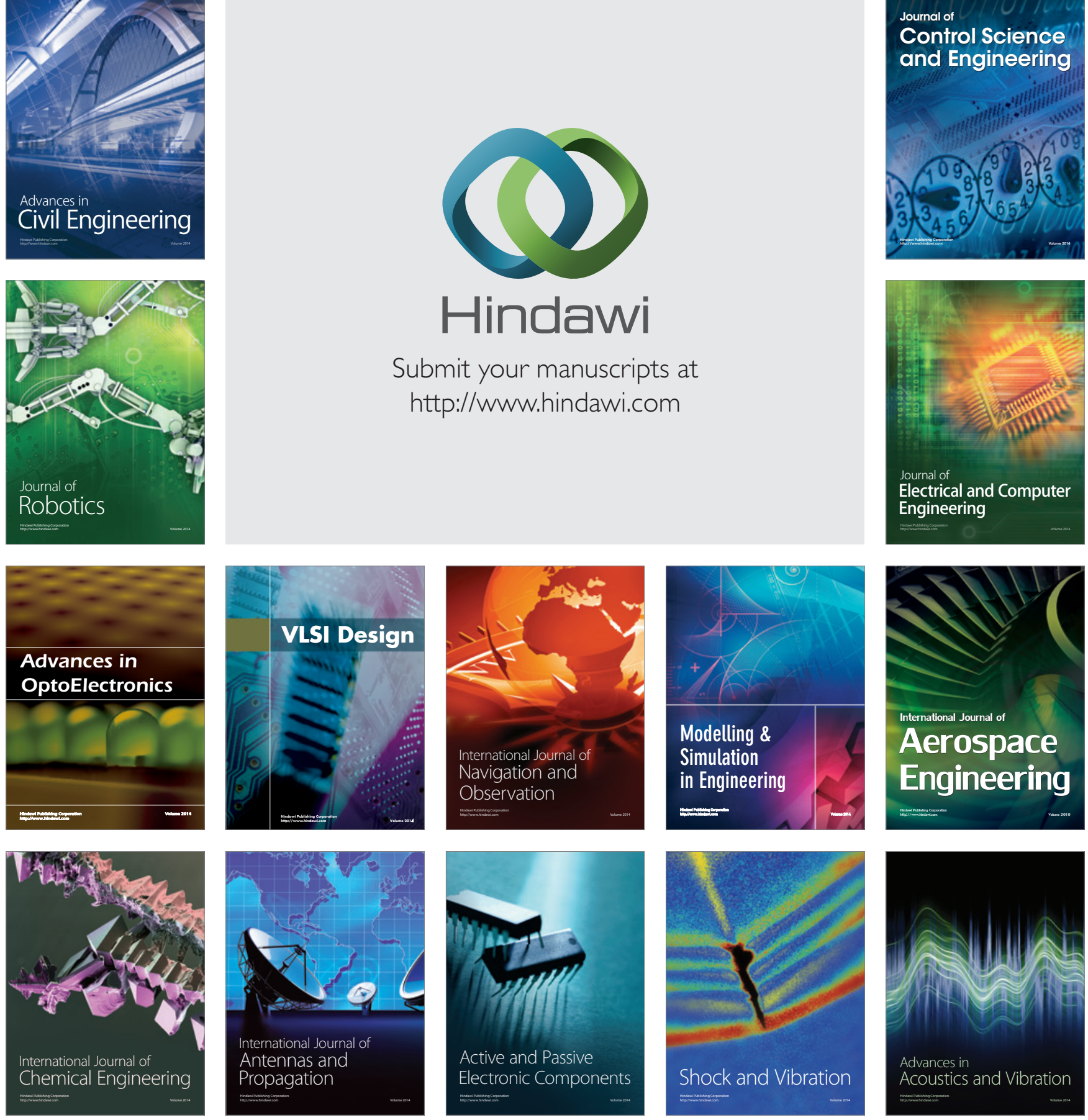